\title{
Towards a Workflow Driven Design for mHealth Devices within Mobile Eye Clinics in Low-Income Settings
}

\author{
Nigel M. Bolster, Member, EMBS, Andrew Bastawrous, and Mario E. Giardini, Member, EMBS
}

\begin{abstract}
Only a small minority of mobile healthcare technologies that have been successful in pilot studies have subsequently been integrated into healthcare systems. Understanding the reasons behind this discrepancy is crucial if such technologies are to be adopted. We believe that the mismatch is due to a breakdown in the relation between technical soundness of the original mobile health (mHealth) device design, and integration into healthcare provision workflows. Quantitative workflow modelling provides an opportunity to test this hypothesis. In this paper we present our current progress in developing a clinical workflow model for mobile eye assessment in low-income settings. We test the model for determining the appropriateness of design parameters of a mHealth device within this workflow, by assessing their impact on the entire clinical workflow performance.
\end{abstract}

\section{INTRODUCTION}

Mobile health (mHealth), the use of mobile technologies within healthcare has shown considerable promise in recent years. It offers an opportunity to make a transformative impact on healthcare delivery, particularly when large populations of at-risk individuals are involved. Low-income settings represent an interesting example as, in such settings, mHealth has the potential to be integrated where current standards are not presently viable due to cost, required specialist skills or infrastructure limitations [1]. Despite their formidable potential and the reported success of pilot studies, relatively few mHealth interventions have subsequently reported effectiveness in multisite trials or have been integrated for use within healthcare systems [2, 3]. We hypothesize that a reason for this apparent contradiction is a breakdown in the transition between successful completion of pilot studies and integration into healthcare provision workflows. We believe that, although these device designs deliver good results with respect to their technological requirements, failure in the adoption into healthcare systems

The Nakuru Eye Disease Cohort Study was jointly funded by the Medical Research Council (MRC) and the Department for International Development (DFID) under the MRC/DFID Concordat agreement and Fight for Sight. Additional funding supporting the study (equipment and field staff) were provided by the International Glaucoma Association and the British Council for the Prevention (BCPB).

The Technology for Eye Health project is funded by the Queen's Diamond Jubilee Trust.

N. M. Bolster and M. E. Giardini are with the Department of Biomedical Engineering, University of Strathclyde, Glasgow, G4 0NW, UK (for correspondence telephone: $\quad+44(0) 1415-48-3142$; e-mail: nigel.bolster@strath.ac.uk).

A Bastawrous is with International Centre for Eye Health, Department of Clinical Research, London School of Hygiene \& Tropical Medicine, London, WC1E 7HT, UK. can be due to a mismatch with the requirements for integration and adoption into the clinical workflow.

In order to test this hypothesis, it is necessary to establish whether or not there is a link between a device's design and the effectiveness and efficacy of the clinical workflow. In assembly line analysis and information technology infrastructure design, the sensitivity of an entire workflow to variation in the parameters of its component processes is commonly assessed using colored Petri Nets. Using these techniques should similarly allow us to assess the sensitivity of a clinical workflow to the engineering parameters of a device.

Ophthalmology has been identified as one specialty of medicine whose quality and capacity in low-income settings could be greatly improved by the adoption of mHealth technologies $[4,5]$. We have therefore chosen to build our workflow model based on data from the Nakuru Eye Disease Cohort Study. This is a recent study that has provided a wealth of information on eye health in a low-income setting. It involved the deployment of a temporary eye clinic, with mHealth devices being trialed in parallel with the reference standard, which consists of diagnostic equipment originally designed for hospital use. Moreover, clinical workflows found in low-income settings generally lack the complex interlinking of their counterparts in high-income settings. Therefore this study constituted a good test case for workflow analysis.

We believe that, were mHealth devices to be fully integrated in the practice, such an adapted mobile clinic could represent a model for mobile clinics deployed as part of country-wide population screening programs, as well as academic research.

To this end, in this paper we present our current progress in developing a clinical workflow model for mobile eye health assessment in low-income settings. This is a clinical workflow which could be transformed by successful incorporation of mHealth devices [5]. We assess the potential use of the model for determining the appropriateness of design parameters for devices within this workflow, by assessing the parameter's impact on the entire clinical workflow performance.

\section{Methods}

\section{A. The Nakuru Eye Disease Cohort Study}

We have used data from the Nakuru Eye Disease Cohort Study (NEDCS) which was a study that conducted assessments of several measures of ocular health of 2185 participants aged 55 years and above in Nakuru, Kenya 


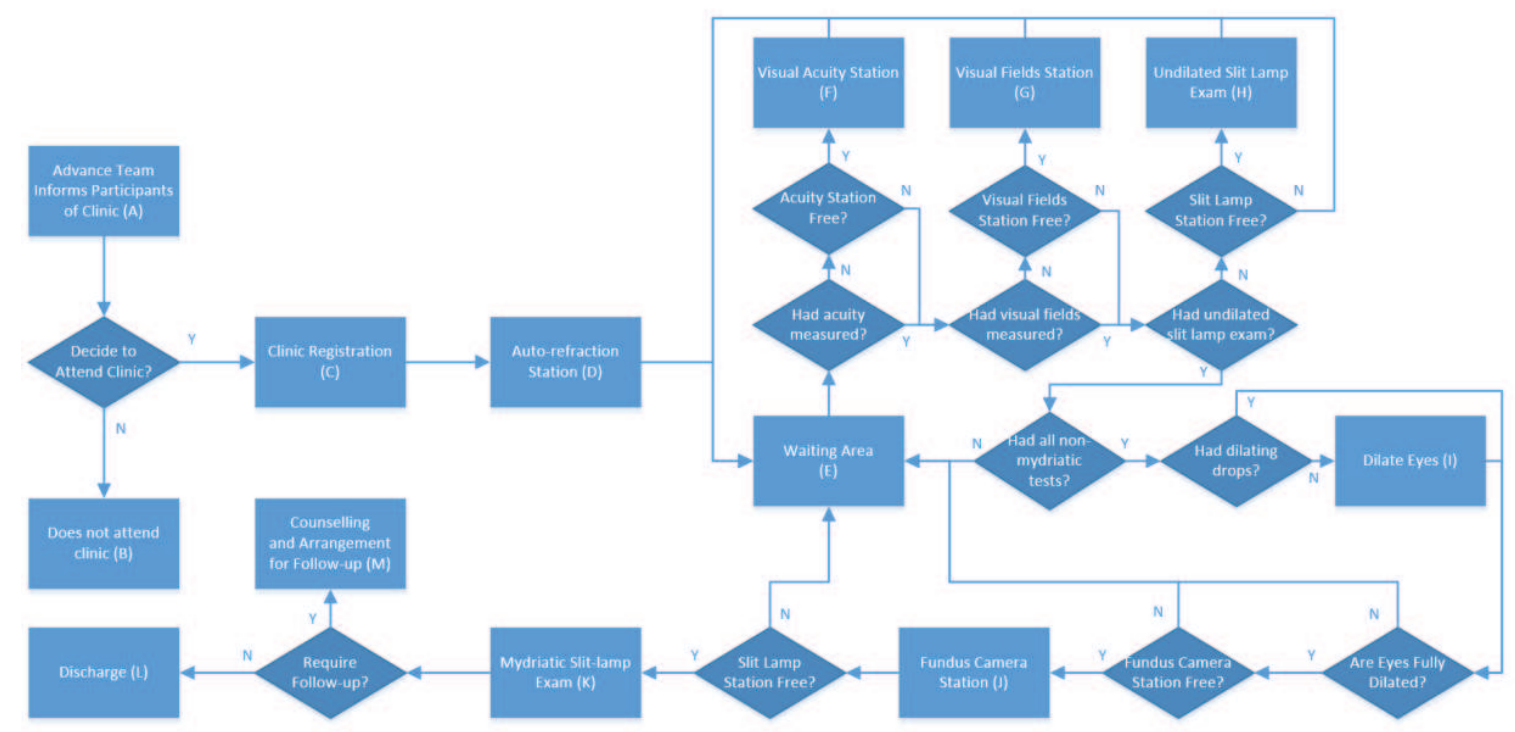

Figure 1. Flow diagram of the clinical workflow for a temporary eye health assessment clinic deployed to low-income settings (the Nakuru Eye Disease Cohort Study [6,7])

during 2013/2014 [6]. Such measures of ocular health included visual acuity (VA), autorefraction, visual fields, slit lamp assessment of the anterior and posterior segment lens grading and fundus photography. Participants were recruited from those selected for a baseline study conducted in 2007/2008 [7]. The study assessed a total of one hundred clusters, each of up to 50 participants, and many of which were in remote rural areas. This involved the deployment of a temporary eye clinic in close proximity to the cluster's participants' most accessible venue. The clinic was equipped with a logmar acuity chart, autorefractor, visual fields test, slit lamp, biomicrocope, and fundus camera. It also trialed two mHealth devices in parallel to these reference standard diagnostic devices, one for the measurement of VA and the other for the measurement of the optic nerve's vertical cup-to-disc ratio (VCDR). Approximately ten clinical workers plus an ophthalmologist staffed the clinic, which received its power from a diesel generator. Study participants were recruited for each cluster by an 'advance team' consisting of two clinical workers plus a local guide. The advance team visited the cluster's location the day before the clinic was due to be deployed to inform participants with respect to the location, time and benefits of attending.

The clinic's workflow is shown in Fig. 1.

\section{B. Workflow Modelling}

The workflow model based on the workflow of the NEDCS was programmed in CPN Tools [8], a program specifically designed for building workflow simulations using the Standard ML of New Jersey (SML/NJ) programming language [9]. Study participants were represented as records consisting of various basic details, such as their willingness to travel to clinic, and of two subrecords containing various indicators of eye health, including those assessed by mHealth devices during the NEDCS, that is VA and VCDR. Each eye health indicator appeared at least twice for each eye, with one representing the actual value for the indicator in question, whilst the second and subsequent appearances represented that measured by the relevant medical device or devices within the workflow.

Medical devices were represented in the model by records containing values describing the eye health indicators they can measure, their minimum unit of measurement and their variance when returned eye health indicators are approximated to a value selected from a normal distribution with a mean equal to the actual indicator value. Additionally the record contained a user learning rate defined as the fractional decrease in variance observed every time the number of measurements previously taken by the user doubled. It was assumed that there were no systematic errors in device measurements. Thus a given measurement was taken from a normal distribution with a given variance centred on the true value. The variance of this distribution would decrease with the number of measurements the user had previously conducted with the device according to Wright's Learning Curve Model, that is $\mathrm{Y}=\mathrm{aX}$, where $\mathrm{Y}$ is the variance, $a$ is the initial variance, $X$ is the number of measurements previously taken and $\mathrm{b}$ is the logarithm of the user learning rate, represented as a decimal, over $\log 2$ [10].

All of the study participants are initially located within a population center (Fig. 1A). The simulation program generates a number of participants, $\mathrm{n}_{\text {cluster }}$, selected from a three-parameter Weibull probability distribution $(\lambda=77.09$, $\mathrm{k}=11.04$, shift $=-46.97)$ where outlying values of $\mathrm{n}_{\text {cluster }}<0$ or $\mathrm{n}_{\text {cluster }}>50$ were rounded to 0 and 50 respectively. Upon generation, each patient record was populated with values for their actual VCDR and willingness to travel to a clinic. VCDRs were independently selected from a gamma distribution ( shape $=7.643$, scale $=0.05189$ ) and willingness to travel to clinic independently selected from a value from 0 to 1000 according to a uniform distribution. Each participant would then progress to clinic registration (Fig. 1C) if their willingness to travel was above a certain threshold value $(180,0.18$ being the proportion of study participants that 
were contacted during the NEDCS but did not subsequently travel to the clinic the following day). If their willingness to travel was below this threshold value then the participant's workflow would terminate with the record's final value being written to the log file.

Having registered, the participants would then progress to a station providing auto-refraction (Fig. 1D). As is the case with every device stations described in this model, only one participant could occupy the station at one moment in time, forcing other participants to queue between the registration desk and auto-refraction. Next, the participants would progress to the waiting area where they would queue until either the VA station (Fig. 1F), visual fields station (Fig. 1G) or undilated slit-lamp examination (Fig. $1 \mathrm{H}$ ) became vacant. After completing this test they would then be returned to the waiting area where they would queue until one of the remaining test became vacant. They would repeat this process until they completed each of these tests after which they would progress to a station for the administering of dilating eye drops (Fig. 1I). Once fully dilated they would progress to a fundus camera station, once vacant, (Fig. 1J) before progressing for a dilated slit-lamp examination (Fig. $1 \mathrm{~K})$. The undilated and dilated slit lamp examinations both involved the use of the same slit lamp and therefore only one of these stations could be occupied by a single participant at any given moment in time. Finally the patient would proceed for counselling and advice on necessary follow-up or treatment if required (Fig. 1M), with the participant's contact details being recorded for the arrangement of an appointment with and transportation to a specialist if follow-up or treatment was agreed upon.

Each of the stations in the clinic was permanently staffed by a clinical worker for the duration of the clinic, with the exception of the slit-lamp which was operated by an ophthalmologist. Clinical workers were modelled as a record

(a)
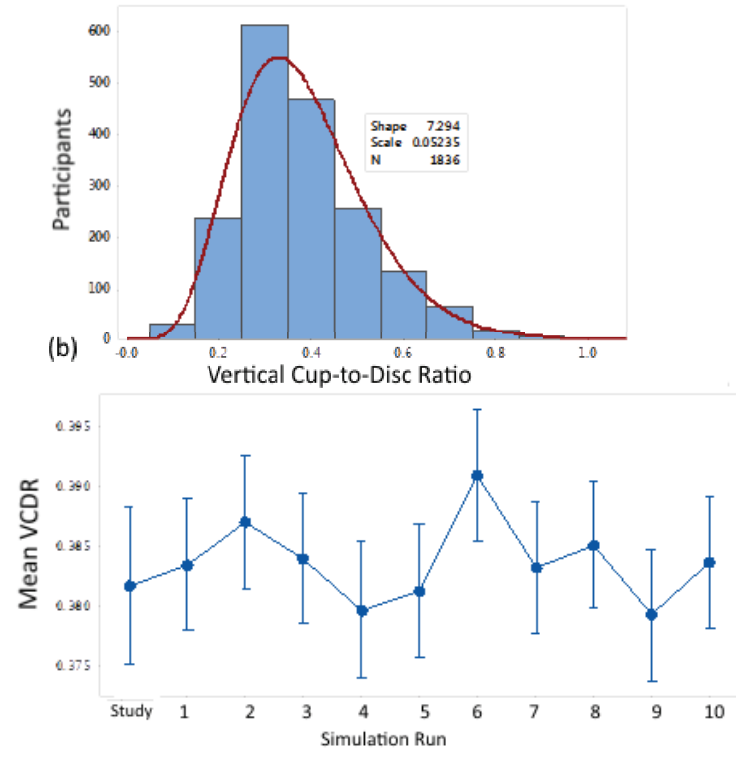

Figure 2. (a) Histogram of the vertical cup-to-disc ratios recorded during the Nakuru Eye Disease Cohort Study. (b) Interval plot of the mean vertical cup-to-disc ratios as found in the study data and in ten simulations. containing the number of times they have used each device in the workflow. The advance team consisted of two clinical workers plus a local guide.

Whether a patient was referred for treatment or not was made based on their exceeding a threshold for any one of the eye health measures, as measured by one or more of the devices. For VCDR this threshold was taken to be a value equal to or greater than 0.7 , in combination with other clinical features.

\section{Statistical Analysis}

All statistical analysis described within was conducting using Minitab 17 Statistical Software (Minitab Ltd., Coventry, U.K.). The type of probability distribution to use for a given generated value within the model was determined by running Minitab's Individual Distribution Identification tool on the appropriate data set from the NEDCS. All analyses of variance (ANOVAs) were one-way and had a $95 \%$ confidence interval unless otherwise stated. Pooled standard deviation was used to calculate the intervals shown on all interval plots.

\section{RESULTS}

\section{A. Simulated Study Samples}

Ten simulations, each including one hundred clusters of up to 50 participants, were run. The generated VCDRs of the right eye were written to a log file for each participant upon termination of their workflow. A two-way ANOVA of the samples suggests that there was no significant difference between the observed sample of VCDRs and those samples which were simulated $(\mathrm{F}=0.01, \mathrm{p}>0.9)$, as shown in Fig. $2 \mathrm{~b}$.

\section{B. Comparison of mHealth Devices' User Learning Rates}

An equal number of simulations were run for two different situations. The workflow for each was identical with the exception of the fundus camera used. This had a user learning rate, with respect to measurement precision, of 0.8 in one scenario compared to 0.95 in the other. The initial variance and minimum unit of measurement were set at typical values of 0.05 and 0.1 respectively in each case. In both cases this simulated instrument represented an mHealth device. Upon completion of each simulation the sensitivity and specificity of the devices' measurement of VCDR was calculated using the generated true VCDR and that measured by the simulated device. The mean sensitivity and specificity of the 0.80 learning rate device was found to be 0.743 and 0.979 respectively. The mean sensitivity and specificity of the 0.95 learning rate device was found to be 0.700 and 0.929 respectively. One-way ANOVAs for the two sets of sensitivities and two sets of specificities are shown in Fig. 3. These showed that the model predicts a statistically significantly improvement in sensitivity and specificity for a fundus camera with a learning rate of 0.80 compared to a similar device with a learning rate of $0.95(\mathrm{~F}$ $=12.02, \mathrm{p}<0.01$ for sensitivities and $\mathrm{f}=397.4, \mathrm{p}<0.001$ for specificities).

\section{Locating of a Device within the Workflow}

We next considered the placement of the mHealth device, with a user learning rate of 0.8 with respect to 


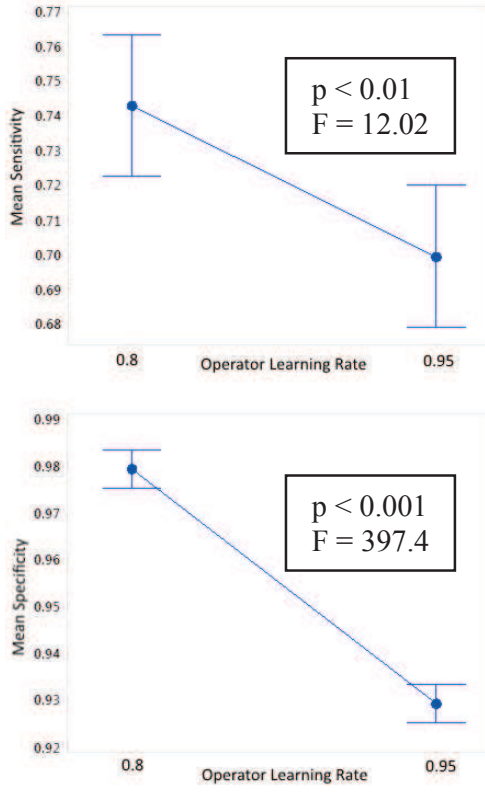

Figure 3. Interval plots for the mean sensitivity (top) and specificity (bottom) achieved for simulated devices measuring participant vertical cup-to-disk ratio. The devices have identical parameters other than the user learning rate with respect to measurement precision.

precision, within the described workflow. We additionally specify that such as device is capable of allowing a clinical worker to measure the VCDR of an undilated eye. A station containing such a device could be added at one of two points in the workflow. Firstly, it could be inserted alongside the other device stations interfacing with the waiting area $(\mathrm{E}$ in Fig. 1) within the clinic. Alternatively the device could be given to the advance team and the VCDR of the participant recorded in their home prior to the clinic. In each case a single user throughout the screening programme with no prior experience of using the device was assumed. Five simulations of each scenario indicated that the placing the device with the advance team consistently achieved screening of a greater number of participants, as those failing to attend the clinic could still be assessed. As a result the mean sensitivity of the device for VCDR based screening was found to have a mean value of 0.847 compared to 0.743 if placed within the clinic. The sensitivity of the entire workflow when the device was clinic based, where no-show positives were also counted as false negatives had a mean value of 0.648 compared to a mean sensitivity of 0.847 for the entire workflow when the device was based with the advance team. A one-way ANOVA of these mean sensitivities (Fig. 4) indicated that the differences were statistically significant $(F=29.47$ and $p=0.001$ for the device and $F=97.47, p<0.001$ for entire workflow). Thus it is clear that such a device would be best located with the advance team as this would involve minimal change to the clinical workflow whilst significantly increasing screening effectiveness.

\section{CONCLUSION}

We have developed a clinical workflow model that allows design parameters of mHealth devices and their relevance to

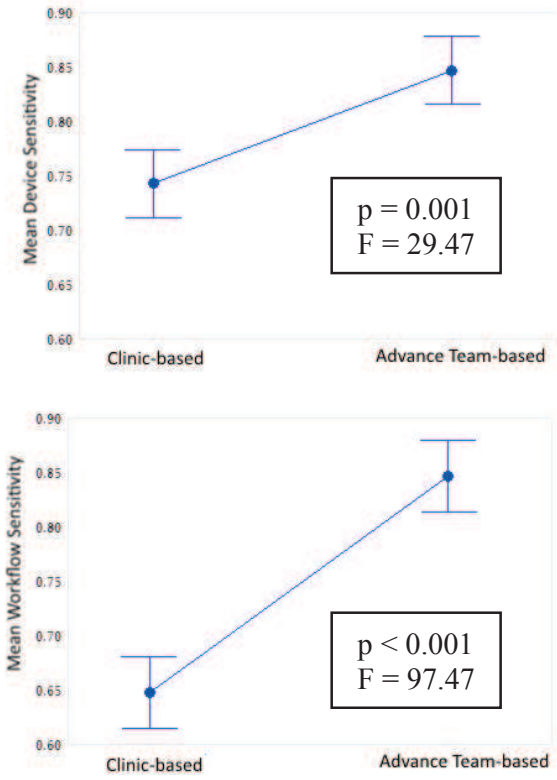

Figure 4. Interval plots for the mean device sensitivity (top) and workflow sensitivity (bottom) achieved for simulated devices inserted at different points in the workflow to measure participant vertical cup-to-disc ratio.

integration into healthcare systems to be assessed. Analysis of this model has been shown to provide useful information relating to a device's user learning rate, with respect to the precision, as a design parameter, and where the device ought to be inserted in a temporary eye disease screening clinic. These results suggest that such a workflow modelling technique may yield useful information in the design of $\mathrm{mHealth}$ devices for integration into healthcare systems.

\section{REFERENCES}

[1] A. Bastawrous and M. J. Armstrong, "Mobile health use in low- and high-income countries: an overview of the peer-reviewed literature," Journal of the Royal Society of Medicine, vol. 106, pp. 130-142, Apr 2013.

[2] C. Z. Qiang, M. Yamamichi, V. Hausman, D. Altman, and I. Unit, "Mobile applications for the health sector," Washington, DC: World Bank, 2012.

[3] M. Tomlinson, M. J. Rotheram-Borus, L. Swartz, and A. C. Tsai, "Scaling up mHealth: where is the evidence?," PLoS Med, vol. 10, p. e1001382, 2013

[4] A. Bastawrous, R. C. Cheeseman, and A. Kumar, "iPhones for eye surgeons," Eye, vol. 26, pp. 343-354, Mar 2012.

[5] N. M. Bolster, M. Giardini, I. Livingstone, and A. Bastawrous, "How the smartphone is driving the eye-health imaging revolution," Expert Review of Ophthalmology, 2014.

[6] A. Bastawrous, W. Mathenge, T. Peto, H. A. Weiss, H. Rono, A. Foster, et al., "The Nakuru eye disease cohort study: methodology \& rationale," BMC ophthalmology, vol. 14, p. 60, 2014.

[7] W. Mathenge, A. Bastawrous, A. Foster, and H. Kuper, "The Nakuru Posterior Segment Eye Disease Study," Ophthalmology, vol. 119, pp. 2033-2039, 2012.

[8] M. Westergaard and T. Slaats, "CPN Tools 4: a process modeling tool combining declarative and imperative paradigms," Automatic Control and Computer Sciences, vol. 47, pp. 393-402, 2013.

[9] A. W. Appel and D. B. MacQueen, "Standard ML of new jersey," in Programming Language Implementation and Logic Programming, 1991, pp. 1-13.

[10] T. P. Wright, "Factors affecting the cost of airplanes," Journal of the Aeronautical Sciences (Institute of the Aeronautical Sciences), vol. 3, 1936. 